\title{
Les sources de pollen et de nectar dans la région de Rimouski, Québec, Canada
}

\author{
J Parent 1, MJ Feller-Demalsy 2, PJH Richard 1 \\ ${ }^{1}$ Université de Montréal, département de géographie, laboratoire de paléobiogéographie \\ et de palynologie, CP 6128 Succ A, Montréal Que, Canada, H3C 3J7 \\ 2 Université du Québec à Rimouski, département de biologie et des sciences de la santé, \\ 300, allée des Ursulines, Rimouski, Que, Canada, G5L 3A1
}

(Reçu le 19 janvier 1990; accepté le 9 juillet 1990)

\begin{abstract}
Résumé - En 1980, l'analyse pollinique des récoltes de pollen et des miels successifs d'une mêrne ruche a été effectuée. Les abeilles ont amassé près de $12,5 \mathrm{~kg}$ de pollen frais sur une période de 3,5 mois. Soixante taxons ont été identifiés par la méthode de tri des pelotes. Les meilleures sources de pollen sont Trifolium hybridum $/ T$ repens, Cornus stolonifera et Salix spp. Des pelotes mixtes, représentant $0,4 \%$ de la récolte totale, ont été trouvées dans $65 \%$ des échantillons analysés. L'analyse pollinique de 13 miels successifs et 3 miels annuels du même rucher a révélé la présence de 42 taxons. Les premiers miels de la saison sont mixtes et composés de plantes printanières, ils sont suivis des miels unifloraux de Rubus idaeus et des miels mixtes annonçant les miels unifloraux de Trifolium hybridum/ $T$ repens. Les miels annuels reflètent l'importance de ces 2 taxons pour le rucher. Notre étude met en évidence la contamination du miel par le pollen récolté par les abeilles. La flore butinée pour le pollen et le nectar est essentiellement la même.
\end{abstract}

miel / pelote de pollen / plante nectarifère / plante pollinifère / analyse pollinique / Canada

\section{INTRODUCTION}

Au Canada, la détermination des sources de pollen utilisées par les abeilles a d'abord été obtenue par la compilation des renseignements fournis par les apiculteurs : Québec (Chabot, 1948), Manitoba (Mitchener, 1948). D'autres travaux couvrant à la fois les États-Unis et le Canada renferment également de nombreux renseignements sur la flore pollinifère (Oertel, 1939, 1967; Robinson et Oertel, 1975; Pellett, 1976; Goltz, 1977; entre autres). En plus de l'observation directe sur le terrain, les sources de pollen utilisées par les abeilles sont également identifiées par l'analyse des pelotes qu'elles rapportent à la ruche: Manitoba (Soehngen, 1971), Ontario (Adams et al, 1978), Alberta (Murrell et Szabo, 1981). Au Québec, mis à part une étude portant sur la composition chimique du pollen recueilli au rucher de Deschambault en 1973 et 1974 (Lamontagne, 1975) et qui présente une chronologie sommaire, on ne dispose que de peu d'informations sur la récolte du pollen par les abeilles. Quant à l'aspect quantitatif de la récolte du pollen, seulement quelques travaux américains présentent des données à ce sujet (Todd et Bishop, 1940; Eckert, 1942).

Par ailleurs, la recherche de l'origine florale des miels du Canada est relativement 
récente et a été effectuée de diverses façons : d'abord, par la détermination de la flore mellifère selon les observations des apiculteurs (essentiellement par les mêmes auteurs que pour la flore pollinifère) ensuite, par l'analyse pollinique des miels (Louveaux, 1966; Feller-Demalsy et Lamontagne, 1979; Feller-Demalsy, 1983; Feller-Demalsy et al, 1987a, 1987b, $1989 a, 1989 b)$ et enfin, par l'analyse des nectars (Adams et al, 1979). Une synthèse portant sur la flore pollinifère et mellifère du Canada incluant la majorité des travaux cités est présentée par Ramsay (1987).

Outre l'apport de données nouvelles sur les sources de pollen au Québec, notre étude permet de déterminer les sources de nectar qui entrent dans la composition des tout premiers miels de la saison, ces miels qui, s'ils ne sont pas utilisés par les abeilles ni extraits rapidement, seront dilués par celui de la grande miellée. En effet, au Canada, l'extraction n'a généralement lieu qu'une fois à l'automne ou 2 fois l'an, à la fin de juillet et à l'automne.

L'Est du Québec constitue, par ailleurs, un cadre d'étude intéressant de par les conditions climatiques qui y règnent. Cette région est caractérisée par un printemps tardif ayant pour effet de réduire d'avril à septembre la durée de la saison apicole. De plus, c'est la première fois à notre connaissance, qu'en Amérique du Nord, une étude parallèle est menée entre ces 2 types de récolte : pelotes de pollen et miel.

\section{MATÉRIEL ET MÉTHODES}

L'expérience a porté sur une ruche faisant partie d'un rucher de 5 unités situé dans la région du Bas Saint-Laurent, dans le comté de Rimouski, à une quinzaine de $\mathrm{km}$ au sud-est de Rimouski, près de Sainte-Blandine (fig 1).

L'expérience s'est déroulée en 1980, du 2 mai (mise en ruche) au 6 septembre, sur une colonie nouvelle d'abeilles de race italienne (Apis mellifera $L$ var ligustica Spinola) introduite dans une ruche neuve (type Langstroth à 10 cadres). Le 14 juin, une seconde hausse à couvain $(C 2)$ était superposée à la première $(C 1)$ et le 24 juin, une hausse à miel $(M)$ et un gardereine complétaient la ruche. Pour éviter les contaminations, les abeilles ont dû bâtir presque tous leurs cadres. Le 6 septembre, la ruche fut dépeuplée de ses abeilles et les hausses entreposées jusqu'à l'extraction du miel.

Des prélèvements de pollen ont été effectués à l'aide d'une trappe à grillage simple (type OAC, Smith et Adie, 1963), quotidiennement du 15 mai au $1^{\text {er }}$ septembre, sauf du 30 mai au 14 juin où la trappe était retirée occasionnellement. Les échantillons étaient ensuite nettoyés, pesés, séchés puis conservés au congélateur. L'efficacité de la trappe a été estimée à près de $10 \%$ (moyenne de 4 mesures) selon la méthode de Synge (1947). Près d'une récolte sur deux (55 sur 97) a été analysée selon la méthode de tri des pelotes (Louveaux, 1954, 1955, 1958a). Les résultats de l'analyse pollinique sont exprimés en pourcentage du poids de chaque taxon sur le poids total (stabilisé à l'air ambiant) de la récolte de la journée (fig 2).

Du 23 mai au 6 septembre, les faces (A et B) de chaque cadre numéroté ont été photographiées hebdomadairement. Les diapositives qui présentaient la plus grande surface de miel operculé en une semaine ont été sélectionnées. Le contour de cette surface était reporté, grandeur nature, sur papier puis sur le cadre correspondant en perçant à la fois le papier et les opercules avec une aiguille. Le secteur était ensuite désoperculé au scalpel et le miel prélevé à l'aide d'une pompe à vide. Toute alvéole anormale était exclue. Le système était abondamment rincé entre chaque prélèvement. Les miels extraits ont été conservés au réfrigérateur jusqu'à leur analyse. Au total, 13 miels successifs (S), couvrant la période du 20 juin (début de l'operculation) au 6 septembre, ont été prélevés. Parmi ceux-ci, des miels contemporains, 2 provenant des faces d'un même cadre (S-10a, S$10 \mathrm{~b})$ et 2 autres extraits de cadres différents ( $S$ $3 a, S-3 b)$, ont été comparés. Trois miels annuels (A) provenant du même rucher et produits en $1978,1979,1980$ ont également été analysés. L'échantillon $\mathrm{A}-80$ provient des 4 ruches entourant la ruche expérimentale.

Le traitement des échantillons de miel a été réalisé sur $15 \mathrm{~g}$ selon les techniques recomman- 
dées par Louveaux et al (1978). La détermination du nombre de grains de pollen a été effectuée avec un hémacytomètre et les résultats reportés sur $10 \mathrm{~g}$ de miel. Les analyses ont porté sur 300 grains de plantes mellifères (sauf $S-10 b$ : 264 grains). Le statut exclusivement pollinitère de Hypericum perforatum n'étant pas clairement établi, cette espèce a été également considérée comme nectarifère. L'appellation florale de chaque miel a été déterminée par l'utilisation des classes de fréquence classiques (Louveaux et al, 1978), telles que reportées à la figure 3.

L'identification des taxons a été précisée selon les règles de Birks (1973). Le type Barbarea vulgaris comprend également : Brassica napus / Brassica rapa et Erysimum cheiranthoides. Le type Amelanchier/Sorbus regroupe A fernaldii, A stolonifera et $S$ americana. Le type Carum carvi inclut aussi Daucus carota. Les Composées Liguliflores sont représentées par le type Taraxacum officinale. Les Aster, Eupatorium et Solidago sont compris dans le type Aster / Solidago. Le type Chrysanthemum leucanthemum inclut également Achillea millefolium. Les espèces comprises dans le genre Malus sont Malus pumila et une série de pommiers cultivés près des résidences. Quant aux Salix, il s'agit principalement de $S$ discolor et de $S$ bebbiana.

Du 30 avril au 25 août, les espèces en fleurs (sauf certaines plantes anémophiles), présentes à l'intérieur et à l'extérieur des 44 quadrats disposés au hasard dans un rayon de $1,5 \mathrm{~km}$ de la ruche (fig 1), ont été recensées une fois par semaine, suivant le même tracé. Au total, 155 espèces de plantes vasculaires ont été identifiées. De ce nombre, $79 \%$ sont des plantes herbacées, $14 \%$ des arbustes ou arbrisseaux et $7 \%$ des arbres. Un spécimen d'herbier et une lame de pollen ont été montés pour fin de contrôle. La vocation principale de chaque aire homogène du territoire est définie sur la figure 1.

Les données météorologiques courantes ont été mesurées quotidiennement près de la ruche (température, précipitations, nébulosité) ou à la station de Rimouski (vélocité moyenne du vent). Ces données sont reportées à la fin du diagramme pollinique (fig 2). D'avril à septembre, la saison apicole de 1980 a été caractérisée par des conditions météorologiques défavorables qui ont été ressenties à travers tout le Québec (Environnement Canada, 1978, 1979, 1980).

\section{RÉSULTATS}

\section{Pollen}

Au total, $1226 \mathrm{~g}$ de pollen frais ont été extraits de la trappe (tableau I). Compte tenu de l'efficacité de la trappe (10\%) et du retrait occasionnel de la trappe, les abeilles ont amassé près de $12,5 \mathrm{~kg}$ de pollen frais sur une période de 3,5 mois.

Au total, 60 taxons répartis en 24 familles et 46 genres ont été identifiés (fig 2). Les proportions présentées dans le tableau II correspondent à la somme des poids de chaque taxon sur le poids total (stabilisé) des échantillons analysés (récolte totale). Trois taxons représentent plus de $10 \%$ de la récolte totale : ce sont Trifolium hybridum $/ T$ repens $(24,6 \%)$, Cornus stolonifera $(19,1 \%)$ et Salix spp avec $10,2 \%$ de la récolte. Treize autres taxons ont fourni $1-10 \%$ du pollen récolté (tableau II) alors que la majorité, soit 44 taxons, a contribué à moins de $1 \%$ de la récolte totale. Dans les récoltes individuelles, 8 taxons sur $60(13,3 \%)$ ont atteint des proportions supérieures à $50 \%$. S'ajoutent à ceux identifiés dans le tableau II, Epilobium angustifolium, présent à proximité de la ruche, et dont l'apport a exceptionnellement atteint $95 \%$ ( 23 juillet), à cause de mauvaises conditions météorologiques. Les autres taxons se classent comme suit : $17(28,3 \%)$ avec plus de $10 \%$ dans au moins une récolte, $18(30,0 \%)$ avec plus de $1 \%$ dans au moins une récolte et $17(28,3 \%)$ n'ayant jamais atteint $1 \%$ dans les récoltes.

Des pelotes mixtes (125 au total), dont la composition détaillée est présentée dans Parent (1983), ont été trouvées dans $65 \%$ des récoltes analysées (fig 2). La proportion des pelotes mixtes dans chaque 


\section{SITE D'ÉTUDE}

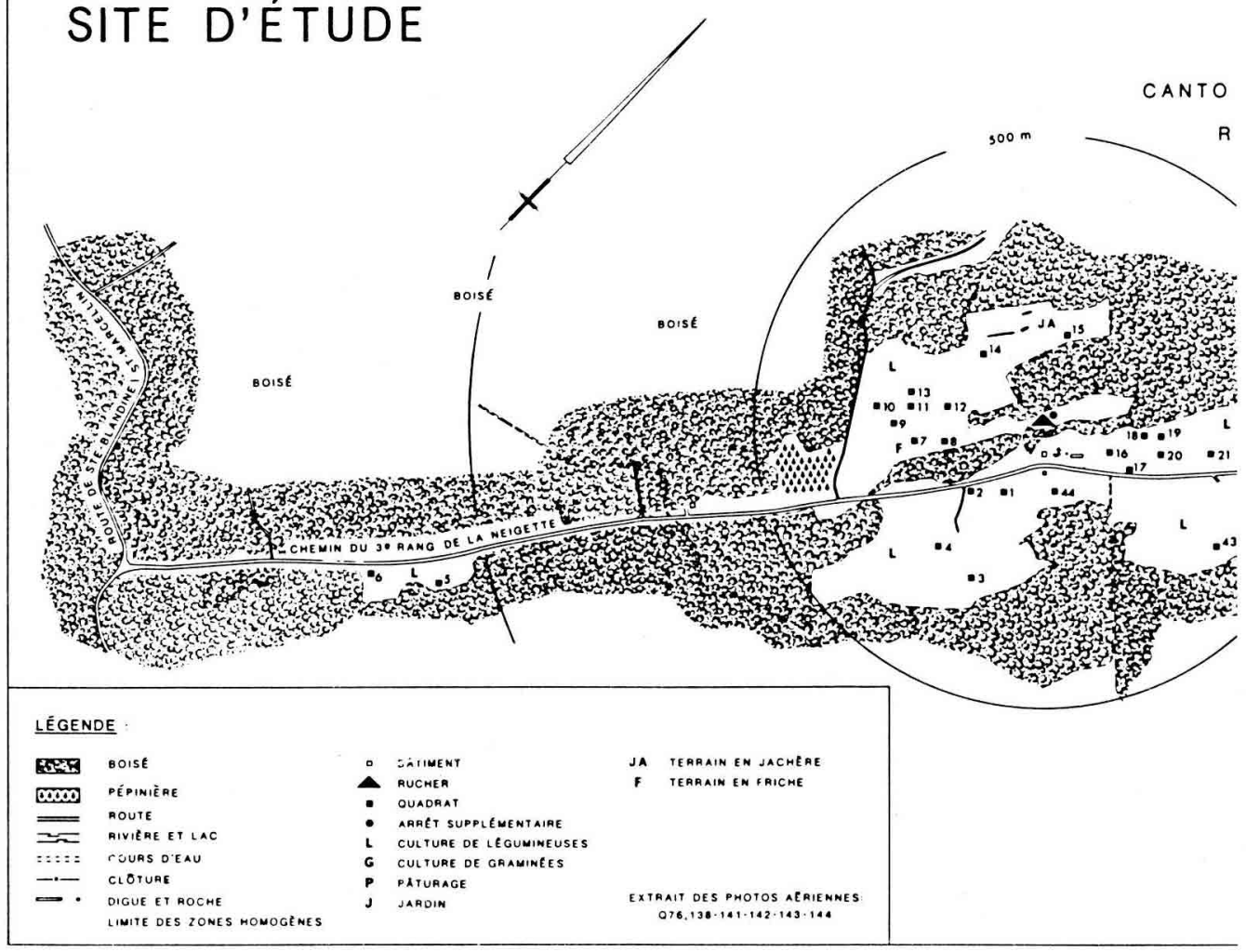

Fig 1. Site d'étude

échantillon varie entre $0,07 \%$ (27 mai) et $2,22 \%$ (12 août); toutefois, leur contribution à la récolte totale ne correspond qu'à $0,38 \%$. Des 36 taxons identifiés dans les pelotes mixtes (tableau III), 15 font partie des espèces les plus visitées ( $>1 \%$ de la récolte totale) et 18 sont classés parmi les espèces les moins fréquentées $(<1 \%$ ). Les autres composantes sont : Malva moschata, Nemopanthus mucronatus (cf) et une troisième, indéterminée. De plus, 3 pelotes de propolis mélangées à du pollen ont été trouvées. Parmi les pelotes mixtes à 2 composantes, observées dans $90 \%$ des cas (109 pelotes), les mélanges pouvaient mettre en présence un arbre et un arbuste $(2,8 \%), 2$ arbustes $(2,8 \%)$, une plante herbacée et un arbuste $(16,5 \%)$ ou 2 plantes herbacées $(78,0 \%)$.

\section{Miel}

La figure 3 présente les résultats de l'analyse pollinique des miels successifs (S) et des miels annuels $(A)$. Les autres rensei- 


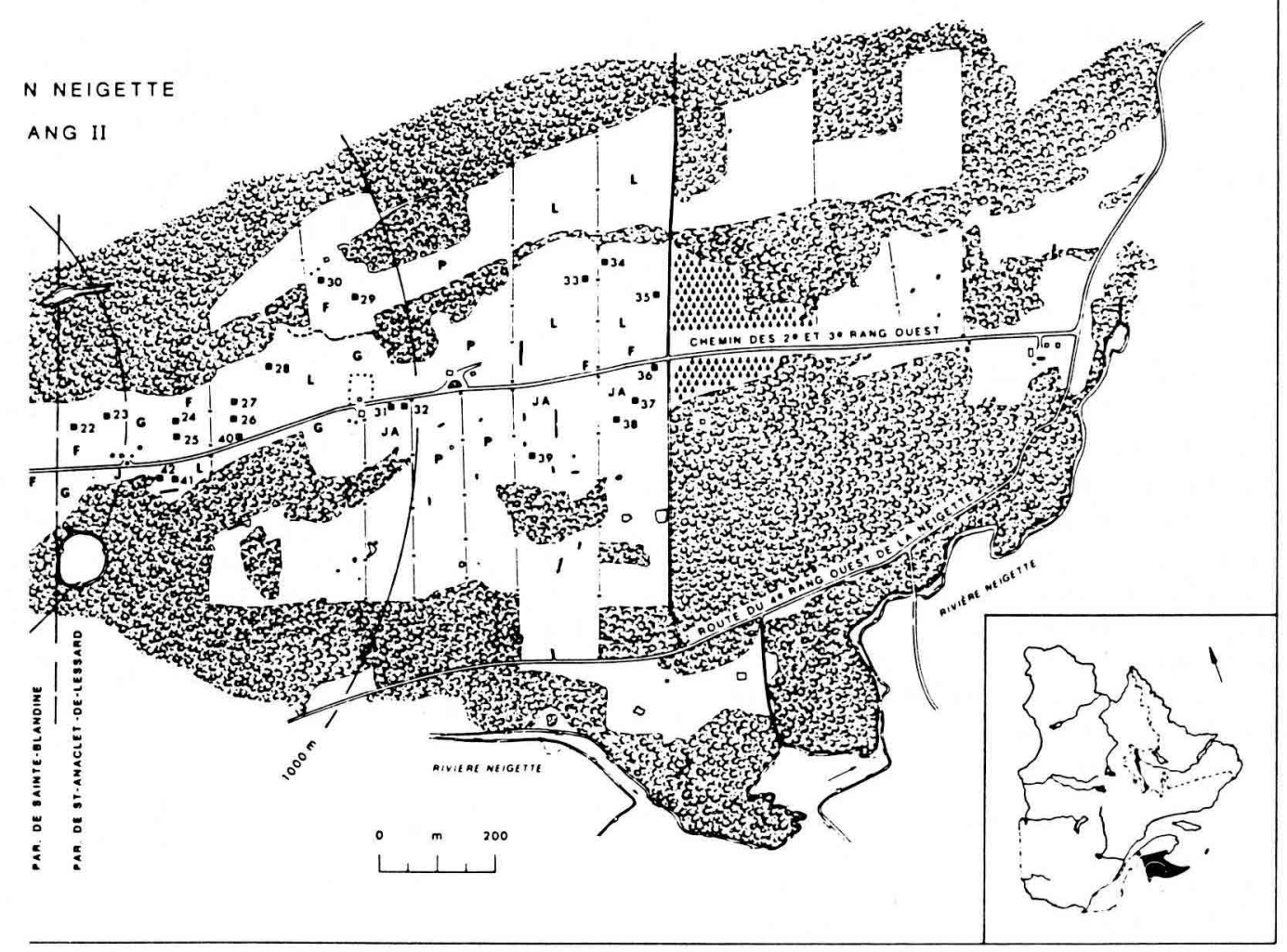

gnements se rapportant à chaque miel sont inscrits au tableau IV. Au total, 42 taxons, dont 34 nectarifères, ont été identifiés dans les miels. Parmi les 10 principales sources de nectar ( $16 \%$ et plus), Rubus idaeus, Trifolium hybridum / $T$ repens et Medicago sativa présentent des cas de dominance.

Le nombre de grains de pollen varie entre 370 et 33148 pour les miels successifs, et entre 2592 et 37222 pour $10 \mathrm{~g}$ de miel pour les miels annuels (tableau IV). La plupart de nos miels se classent donc parmi les miels pauvres (moins de 20000 grains). Seuls les échantillons S-3a et A80 se trouvent parmi les miels à teneur moyenne (entre 20000 et 100000 grains). II n'existe pas de corrélation significative entre la concentration pollinique des miels étudiés et la quantité de miel extrait ( $C$ et $P$, tableau IV) $r=0,3245 ; P>0,05$; ni entre la concentration pollinique et la durée de remplissage des alvéoles $(C$ et $J$, tableau IV) $r=-0,4145 ; P>0,05$. La concentration pollinique d'un miel semble donc indépendante de la quantité prélevée et du temps mis à sa fabrication.

Le nombre de formes nectarifères varie de 6 à 18 chez les miels successifs et de 16 à 21 pour les miels annuels (tableau IV). Le nombre de formes d'abord élevé au printemps, diminue ensuite pendant la pé- 
riode de transition entre les plantes printanières et le trèfle, augmente durant la grande miellée (sauf $\mathrm{S}-8$ ), puis diminue graduellement jusqu'à la fin de la saison.

\section{DISCUSSION}

\section{Pollen}

Le rendement de nos abeilles, $3,6 \mathrm{~kg} /$ mois, se situe au-delà ou près des moyennes observées par d'autres auteurs (Todd et Bishop, 1940 : 1,3-1,9; Hirschfelder, $1951: 2,5-4,7$; Louveaux, $1955: 5,5$; McLellan, $1976: 2,7-7,5)$. Ce rendement est remarquable, du fait qu'il s'agit d'une colonie nouvelle soumise au stress de la trappe, des nombreux rayons à construire, de la courte période d'échantillonnage et des conditions météorologiques défavorables de l'année 1980.

Les meilleures récoltes (22 juin : $55,4 \mathrm{~g}$, 14 juillet : $54,7 \mathrm{~g}, 19$ juin : $45,0 \mathrm{~g}$ ) ont été observées lors de journées chaudes, ensoleillées et peu venteuses, à la suite de 1 ou 2 journées de mauvais temps. A l'opposé, des récoltes nulles (26 mai, 6 juillet, 9 août) ont été obtenues alors que de basses températures, des précipitations, de forts vents et une nébulosité élevée étaient enregistrés. On peut également imputer aux mauvaises conditions météorologiques, la baisse sensible de la quantité de pollen récolté par les abeilles en juillet (tableau I).

Peu de taxons (16) contribuent à un apport quantitatif important (tableau II) alors que la majorité (44) ne participent qu'à une petite fraction de la récolte. II semblerait toutefois qu'une telle variété dans le régime alimentaire des abeilles soit indispensable à la santé de la colonie (Louveaux, 1958a). Les taxons qui ont le plus
Tableau I. Poids frais des récoltes de pollen.

\begin{tabular}{lcrr}
\hline Mois & $\begin{array}{c}J d e \\
\text { prélèvement }\end{array}$ & $\begin{array}{c}\text { Poids frais } \\
(g)\end{array}$ & $\begin{array}{c}\text { Moyenne } \\
(g / \text { mois })\end{array}$ \\
\hline Mai & 20 & 179,22 & 8,96 \\
Juin & 21 & 385,03 & 18,33 \\
Juillet & 31 & 276,25 & 8,91 \\
Août & 31 & 381,03 & 12,29 \\
Septembre & 1 & 4,84 & - \\
& & & \\
Total & 104 & 1226,37 & 11,79 \\
\hline
\end{tabular}

contribué à la récolte totale (Trifolium hybridum IT repens, Cornus stolonifera et Salix spp) ont également dominé (plus de $50 \%$ ) dans les récoltes individuelles.

La proportion de pelotes mixtes par rapport à la récolte totale $(0,4 \%)$ se situe

Tableau II. Contribution à la récolte totale de pollen des taxons prédominants (plus de $1 \%$ ).

\begin{tabular}{|c|c|}
\hline Taxons prédominants & $\%$ \\
\hline $\begin{array}{l}\text { * Trifolium hybridum (c) / T repens (c) } \\
\text { * Cornus stolonifera } \\
\text { * Salix spp } \\
\text { *ype Taraxacum officinale } \\
\text { Brassica kaber } \\
\text { Barbarea vulgaris } \\
\text { Impatiens capensis } \\
\text { Solidago canadensis } \\
\text { Trifolium pratense (c, en partie) } \\
\text { Acer saccharum } \\
\text { Rubus idaeus } \\
\text { *ypericum perforatum } \\
\text { * Type Carum carvi } \\
\text { Acer spicatum } \\
\text { Fragaria virginiana } \\
\text { Acer rubrum }\end{array}$ & $\begin{array}{r}24,58 \\
19,10 \\
10,19 \\
8,55 \\
5,10 \\
4,89 \\
3,78 \\
2,41 \\
2,40 \\
2,26 \\
1,78 \\
1,68 \\
1,54 \\
1,51 \\
1,12 \\
1,09\end{array}$ \\
\hline
\end{tabular}

* : Plus de $50 \%$ dans au moins une récolte; c : espèce cultivée. 
parmi les plus faibles obtenues par d'autres auteurs : 0,1\%-7\% (Betts, 1920, 1935; Percival, 1947; Maurizio et Kollmann, 1949; Maurizio, 1953; Free, 1963). Tant les espèces les plus visitées que celles qui le sont moins sont associées dans les pelotes mixtes; le pollen de plantes prédominantes comme Trifolium hybridum / $T$ repens, type Taraxacum officinale, Brassica kaber et Salix spp y est cependant fréquent (respectivement $47,2 \%, 28,0 \%, 26,4 \%$ et $12,8 \%$; tableau III). Bien que les mélanges aient mis en présence des plantes dont les fleurs étaient situées à des hauteurs différentes, la majorité des associations impliquaient des fleurs de même niveau, principalement des plantes herbacées, reflétant vraisemblablement la flore accessible aux abeilles. En effet, les plantes herbacées dominaient le territoire, suivaient ensuite les arbustes puis les arbres.

Sur une possibilité de 155 plantes vasculaires inventoriées, 60 formes polliniques ont été identifiées dans les récoltes de pollen. Sauf Heracleum maximum (non localisée) et Potentilla argentea, toutes les autres espèces étaient représentées à l'intérieur d'un rayon de $1 \mathrm{~km}$ du rucher. Seuls, Brassica kaber, Arctium, Syringa, Brassica napus / B rapa, Solidago graminifolia et Melilotus ne se trouvaient pas à moins de $500 \mathrm{~m}$. L'abondance et la proximité des individus de Trifolium hybridum ainsi que l'étendue de sa floraison en ont fait la source de pollen la plus exploitée par les abeilles ( $25 \%$ de la récolte totale). En fait, après la fenaison (25 août), on assistait à une diminution très sensible de l'apport pollinique (fig 2). La deuxième espèce la plus visitée fut Cornus stolonifera (19\%); son succès s'explique par sa floraison intermédiaire entre celle des plantes printanières et celle du trèfle. Salix spp vient en troisième position avec $10 \%$, illustrant l'importance des besoins de la colo- nie en début de saison. Les autres espèces choisies par les abeilles étaient, pour la plupart, nectarifères et faisaient partie de la flore indigène ou naturalisée.

Quelques formes non mellifères anémophiles (Rumex, Typha, Graminées, Populus, Cypéracées, Chenopodium) ont été identifiées dans les récoltes mais leur apport reste toujours faible $(<3 \%$ dans la majorité des cas) et la somme de leur contribution ne dépasse pas $0,2 \%$ de la récolte totale. La grande quantité de pollen produite par ces plantes semble attirer les abeilles et ce, même si leurs fleurs ne sont pas adaptées à l'entomophilie (Louveaux, 1958a).

Plusieurs espèces recensées sur le terrain ont été ignorées par les abeilles. Bien que la rareté (Coptis groenlandica) ou l'éloignement relatif (Ribes glandulosum) puissent en être la cause, l'absence dans les récoltes de pollen d'espèces abondantes à proximité du rucher (Galium mollugo, Myosotis arvensis) serait vraisemblablement due à la floraison d'espèces concurrentes, notamment le trèfle hybride.

\section{Miel}

Compte tenu du chevauchement de certains échantillons, on peut résumer comme suit l'évolution de la composition du miel en 1980 (fig 3). La première période, assez courte et se terminant à la fin juin, est caractérisée par des miels mixtes de plantes printanières (S-1, S-2). Elle est suivie d'une période dominée par Rubus idaeus jusqu'à la fin de juillet (S-3 à $S-5$ ). Vient ensuite une période de transition, qui s'étend aussi sur près d'un mois, entre les miels de Rubus idaeus et ceux de Trifolium hybridum / $T$ repens (S-7 à S-9); seul S-6 fait exception à cette règle. Enfin, de la mi-août jusqu'en septembre, on retrouve 
Tableau III. Taxons présents dans les pelotes mixtes.

\begin{tabular}{lrrlrrr}
\hline \multicolumn{1}{c}{ Taxons } & $N$ & $F$ & & Taxons & \\
& & & & & \\
\hline & 1 & 0,8 & Acer spicatum & 3 & 2,4 \\
Acer rubrum & 1 & 0,8 & Arctium minus & 10 & 8,0 \\
Aralia nudicaulis & 3 & 2,4 & Barbarea vulgaris & 7 & 5,6 \\
Aster umbellatus & 33 & 26,4 & Brassica napus / B rapa & 12 & 9,6 \\
Brassica kaber & 3 & 2,4 & Chrysanthemum leucanthemum & 1 & 0,8 \\
Carum carvi (type) & 10 & 8,0 & Cornus canadensis & 2 & 1,6 \\
Cirsium spp & 5 & 4,0 & Epilobium angustifolium & 6 & 4,8 \\
Cornus stolonifera & 2 & 1,6 & Fragaria virginiana & 11 & 8,8 \\
Eupatorium (cf) & 8 & 6,4 & Impatiens capensis & 2 & 1,6 \\
Hypericum perforatum & 1 & 0,8 & Malva moschata \# & 1 & 0,8 \\
Lonicera canadensis & 1 & 0,8 & Oenothera perennis & 1 & 0,8 \\
Nemopanthus mucronatus (cf) & 2 & 1,6 & Ranunculus acris & 1 & 0,8 \\
Prunus pensylvanica & 8 & 6,4 & Salix spp & 16 & 12,8 \\
Rubus idaeus & 3 & 2,4 & Silene cucubalus & 7 & 5,6 \\
Sambucus pubens & 2 & 1,6 & Solidago graminifolia & 1 & 0,8 \\
Solidago canadensis & 8 & 6,4 & Taraxacum officinale (type) & 35 & 28,0 \\
Stellaria graminea & 2 & 1,6 & Trifolium hybridum / Trepens & 59 & 47,2 \\
Thalictrum pubescens & 5 & 4,0 & Indéterminé & 1 & 0,8 \\
Trifolium pratense & 3 & 2,4 & & & \\
Propolis + pollen & & & & & \\
& & & & &
\end{tabular}

$\mathrm{N}$ : nombre de pelotes mixtes comprenant le taxon; $\mathrm{F}$ : fréquence dans les pelotes mixtes; \# : absent des pelotes monospécifiques.

des miels dominants de Trifolium hybridum $T$ repens (S-10, S-11), le dernier (S11) présentant une co-dominance avec Medicago sativa.

Bien que l'association Rubus et Trifolium hybridum / $T$ repens se retrouve dans les 3 miels annuels (fig 3), l'importance relative de ces 2 espèces semble être reliée aux conditions météorologiques. La saison apicole 1979, plus chaude que les autres, mais plus pluvieuse qu'en 1978 (Environnement Canada, 1978, 1979, 1980) aurait favorisé la production de nectar de Trifolium repens (Pellett, 1976; Goltz, 1977) et, vraisemblablement, celle de Trifolium hybridum également $(54,0 \%$ contre $43,0 \%$ en 1978 et $28,3 \%$ en 1980 pour les 2 espèces). Par ailleurs, il semble que la sécrétion de nectar de Rubus idaeus serait moins affectée par des conditions plus dif- ficiles (Morse, 1980), ce qui expliquerait l'importance de sa contribution dans le miel de 1980 (42,0\% comparativement à $16,0 \%$ en 1978 et à $14,3 \%$ en 1979 ).

Les miels operculés la même semaine et provenant des 2 côtés d'un même cadre (S-10a, C1-3A; S-10b, C1-3B) sont plus semblables de par leur composition, leur concentration pollinique et le nombre de formes que 2 autres miels, operculés la même semaine, mais extraits de cadres différents (S-3a, C2-VA; S-3b, C1-VIA). Ces données semblent indiquer que les abeilles entreposent le nectar de façon systématique (côtés d'un même cadre) plutôt que de façon désordonnée.

La présence dans le miel d'espèces anémophiles ou entomophiles sans nectaires floraux est fort courante, toutefois 
Tableau IV. Caractéristiques des miels.

\begin{tabular}{|c|c|c|c|c|c|c|c|}
\hline$M$ & $\mathrm{H}$ & $D$ & $J$ & $P$ & C & FN & FT \\
\hline$S-1$ & C1 & $06-06$ & 14 & 103,97 & 8333 & 12 & 13 \\
\hline S-2 & C1 & $20-26$ & 9 & 209,96 & 2407 & 17 & 20 \\
\hline$s-3 a$ & $\mathrm{C} 2$ & $29-06$ & 8 & 124,05 & 33148 & 9 & 9 \\
\hline$S-3 b$ & C1 & $29-06$ & 8 & 62,36 & 7036 & 6 & 6 \\
\hline$s-4$ & $\mathrm{C} 2$ & 29-06 & 14 & 169,84 & 4629 & 7 & 8 \\
\hline S-5 & $\mathrm{C} 2$ & 07-07 & 20 & 150,12 & 15555 & 14 & 19 \\
\hline$S-6$ & $M$ & $\# \quad 20-07$ & 28 & 17,13 & 925 & 18 & 22 \\
\hline s-7 & $M$ & \# 20-07 & 35 & 40,63 & 740 & 17 & 21 \\
\hline$S-8$ & $M$ & $\# 20-07$ & 41 & 32,59 & 1666 & 15 & 17 \\
\hline S-9 & $M$ & $\# 20-07$ & 48 & 64,43 & 1666 & 16 & 18 \\
\hline$S-10 a$ & C1 & $17-08$ & 15 & 49,02 & 370 & 14 & 17 \\
\hline$S-10 b$ & C1 & $17-08$ & 15 & 69,07 & 370 & 15 & 18 \\
\hline S-11 & $\mathrm{C} 2$ & $24-08$ & 13 & 22,81 & 3333 & 9 & 9 \\
\hline$A-78$ & & & & & 2592 & 16 & 18 \\
\hline A-79 & & & & & 10555 & 21 & 25 \\
\hline A-80 & & & & & 37222 & 16 & 18 \\
\hline
\end{tabular}

$M$ : numéro du miel; $H$ : hausse d'origine; $D$ : date de début du remplissage des alvéoles; $J$ : durée approximative (j) du remplissage des alvéoles; $P$ : poids $(\mathrm{g})$ de miel extrait $( \pm 0,04 \mathrm{~g}) ; C$ : concentration pollinique (grains $/ 10 \mathrm{~g}$ de miel); $F N$ : nombre de formes nectaritères; $F T$ : nombre total de formes; \# : nectar dans quelques alvéoles dès le 7 juillet.

les proportions élevées de Sambucus pubens (S-1 : 29,2\%) et d'Anemone canadensis (S-5: $86,2 \%$ ), 2 espèces activement butinées par les abeilles (fig 2), et les fortes concentrations polliniques (S-1: 8 333 grains; S-5 : 15555 grains) seraient vraisemblablement dues à une contamination à l'intérieur de la ruche par les jeunes abeilles qui se nourrissent de pollen et qui placent le nectar dans les alvéoles (Louveaux, 1958b). L'échantillon S-3a également caractérisé par une concentration pollinique élevée (33 148 grains) refléterait une contamination du même type. II s'agirait cette fois du pollen de plantes nectarifères, en l'occurrence Rubus idaeus $(55,7 \%)$ et Cornus stolonifera $(41,0 \%)$, alors qu'aucun des 7 autres taxons n'at- teint $1 \%$. Notons que tous ces miels proviennent des hausses à couvain donc, à proximité des réserves de pollen.

Par ailleurs, la présence dans les échantillons de la hausse à miel d'espèces dont la floraison était terminée bien avant sa mise en place (24 juin) prouve sans conteste l'existence d'une contamination. Tel est le cas du saule dont la dernière floraison remonte au 12 juin (fig 2), alors que des proportions de $10,3-40,0 \%$ étaient trouvées dans les miels S-6 à S-9 (M). La concentration pollinique de ces miels demeure cependant relativement faible (7401666 grains). Le transfert de pollen à l'intérieur de la ruche par simple contact entre les abeilles (de Grandi-Hoffman et al, 
1986) pourrait ici expliquer la contamination du miel. Une abeille pourrait ainsi transporter le pollen de plantes dont la floraison est déjà terminée. Tel est le cas également des miels S-10a et S-10b (C1) où des grains de plantes printanières (exemple, Acer, Prunus) étaient observés alors que les floraisons étaient terminées le 17 août (début du remplissage). Bien que des contaminations aient été relevées tant dans les hausses à couvain que dans la hausse à miel, nous croyons comme Louveaux (1958b) que leur importance dépend de la proximité des réserves de pollen.

Une partie seulement de la végétation, soit 42 taxons sur 155 plantes vasculaires, a été identifiée par l'analyse pollinique des miels. Sauf Rhamnus (S-10a) et Spiraea (A-78), les autres espèces nectarifères ont toutes été aperçues durant les inventaires. Bien que la plupart des espèces nectarifères identifiées dans les miels aient été trouvées à moins de $500 \mathrm{~m}$ du rucher, quelques-unes (Brassica kaber, Arctium minus et Syringa vulgaris) étaient représentées à l'intérieur d'un rayon de 1 $\mathrm{km}$ de la ruche. Les taxons non nectarifères retrouvés dans le miel sont des taxons anémophiles qui n'apparaissent pas dans les inventaires de végétation (Ambrosia : 1 grain; cf Callitriche : 1 grain) ou dont le pollen a été activement butiné (Rumex, Graminées, Cypéracées, fig 2). Leur présence dans le miel résulterait respectivement de l'action du vent et d'une contamination par les abeilles. Cette dernière éventualité expliquerait, comme nous l'avons vu, la présence dans le miel de taxons entomophiles (ex Anemone, Sambucus) butinés exclusivement pour leur pollen (Ramsay, 1987). De telles contaminations ont également été relevées dans les nectars (Adams et al, 1979).

\section{Pollen et miel}

La plupart des espèces identifiées dans les miels sont également présentes dans les récoltes de pollen (tableau V). Parmi les espèces qui ont été butinées exclusivement pour leur pollen ( $P$ seulement), plusieurs sont également nectarifères. Leur absence dans le miel peut être attribuée à leur faible représentation sur le terrain (Oenothera perennis), à leur éloignement relatif du rucher (Potentilla argentea), à des conditions météorologiques ne favorisant pas la production de nectar (Melilotus) ou encore, à la floraison d'espèces concurrentes. D'autres espèces, non mellifères, sont à la fois communes aux récoltes de pollen et aux miels ( $P$ et $*$ ). L'intervention des abeilles à l'intérieur de la ruche expliquerait les forts pourcentages observés dans certains cas. Parmi les espèces visitées exclusivement pour leur nectar ( $M$ seulement), Medicago sativa est de loin la plus importante. L'attrait qu'exerce le nectar de cette espèce, et à l'opposé, le désintéressement total des abeilles pour son pollen est confirmé ici. Nos abeilles lui ont donc préféré le pollen d'espèces concurrentes, notamment Trifolium hybridum.

Mis à part le trèfle hybride, cultivé en grande partie, les abeilles ont surtout butiné des plantes de la flore indigène ou naturalisée. La variété florale qu'offraient les aires naturelles, même de petites superficies, face à l'étendue des terres cultivées, a été un facteur déterminant dans le choix des abeilles. L'étude comparative des récoltes de pollen et de miel a permis la reconstitution de l'ensemble de la flore apicole utilisée par les abeilles. Bien que certaines espèces aient été sélectionnées uniquement pour leur pollen ou leur nectar, la flore visitée par les abeilles est essentiellement la même. 


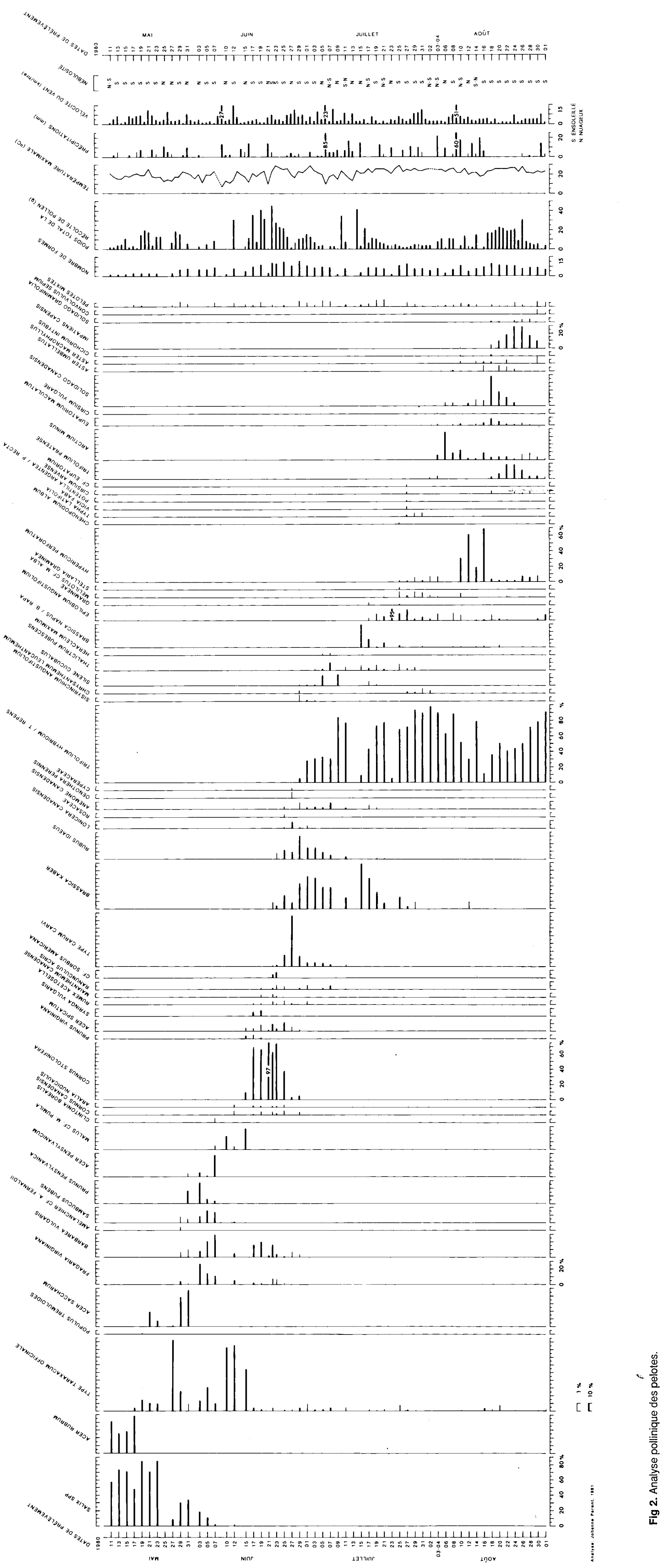




\begin{tabular}{|c|c|}
\hline & $\begin{array}{l}\text { Type Barbarea vulgaris } \\
\text { Salix spo. }\end{array}$ \\
\hline & Typo Taraxacum olficinale \\
\hline & Acer pensyivanicum \\
\hline & Fraparia virginlana \\
\hline & Rubus c1. A. pubescons \\
\hline & Acer saccharum \\
\hline & Tritolium hybridum/t. repens \\
\hline & Type Amelanchier/Sorbus \\
\hline & Prunus pensylvanica \\
\hline & flubus idaeus \\
\hline & Tritolium pratense \\
\hline & Acer spicalum \\
\hline & Cornus stolonitera \\
\hline & Cornus cenadensis \\
\hline & Acer rubrum \\
\hline & Type Carum carvi \\
\hline & Brassica kaber \\
\hline ? & Malus spp. \\
\hline & Syringa vulgaris \\
\hline & Medicago sativa \\
\hline & Victa cracca \\
\hline & Silene cucubalus \\
\hline & Ranunculus acris \\
\hline & Type Chrysanthomum leucanthemum \\
\hline & Hypericum perioratum \\
\hline & Typo Astar/Solidago \\
\hline & Arctium minus \\
\hline & Epilobium angustitolium \\
\hline & Clintonia/Maianthemum \\
\hline & Prunus virginiana \\
\hline & Impatiens capensis \\
\hline & Rhamnus ct. $R$. alnitolia \\
\hline & Spiraea ct. S. Iatifolia \\
\hline & Sambucus pubens \\
\hline & Rumex acetosella \\
\hline & Cyperaceas \\
\hline & Anemone canadensis \\
\hline & Thalictrum pubescens \\
\hline & Ambrosia c1. A. artemisitolia \\
\hline & Gramineae \\
\hline & cl. Callitriche \\
\hline & Indeterminables \\
\hline
\end{tabular}

Classes de trequence
Q 16-45* pollen diaccompagnement

O $3-15 x$ pollen isole important

- Lax pollen isolé rare

pour les plantes meltiferes $\otimes \otimes \cdot 0 \cdot 0.000$.

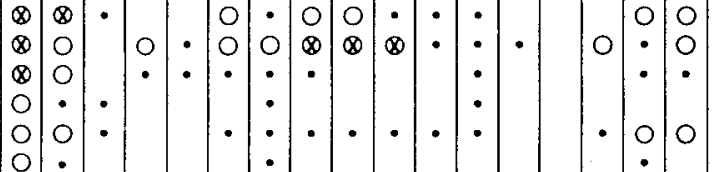

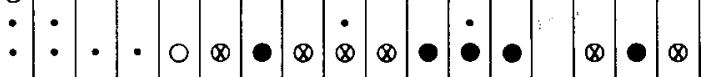

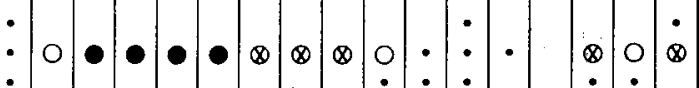

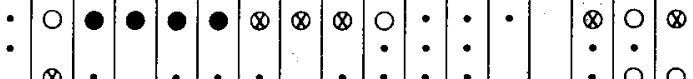

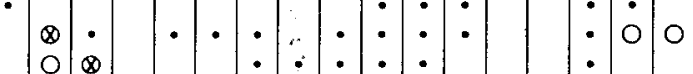

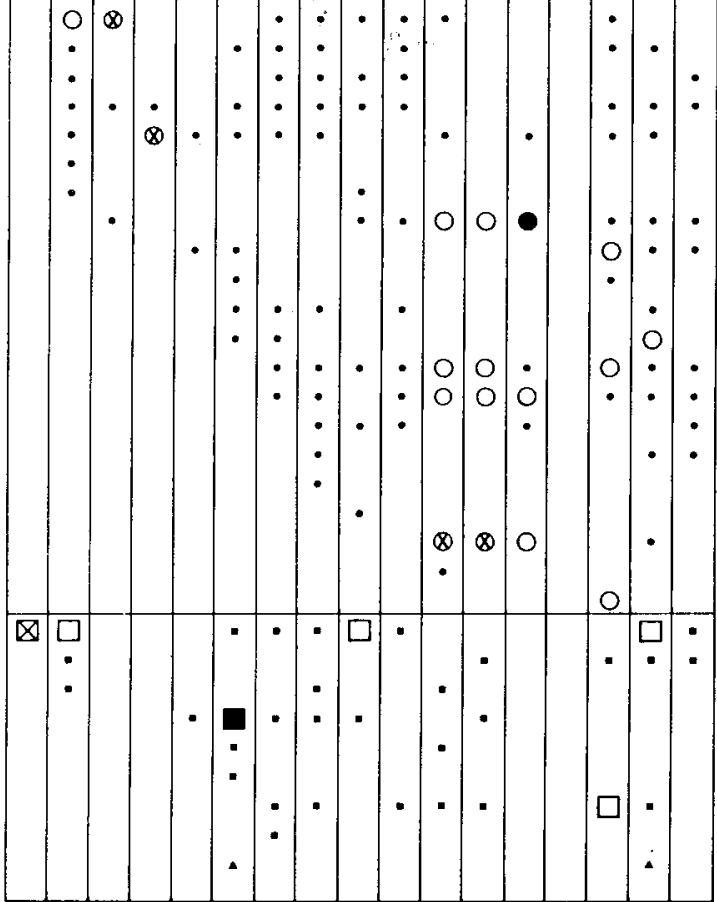

Les grains de pollen indéterminables $(<3 \%)$ sonl représentés par : *

Fig 3. Spectre pollinique des miels. 
Tableau V. Taxons présents dans les récoltes de pollen $(P)$ et dans les miels (M).

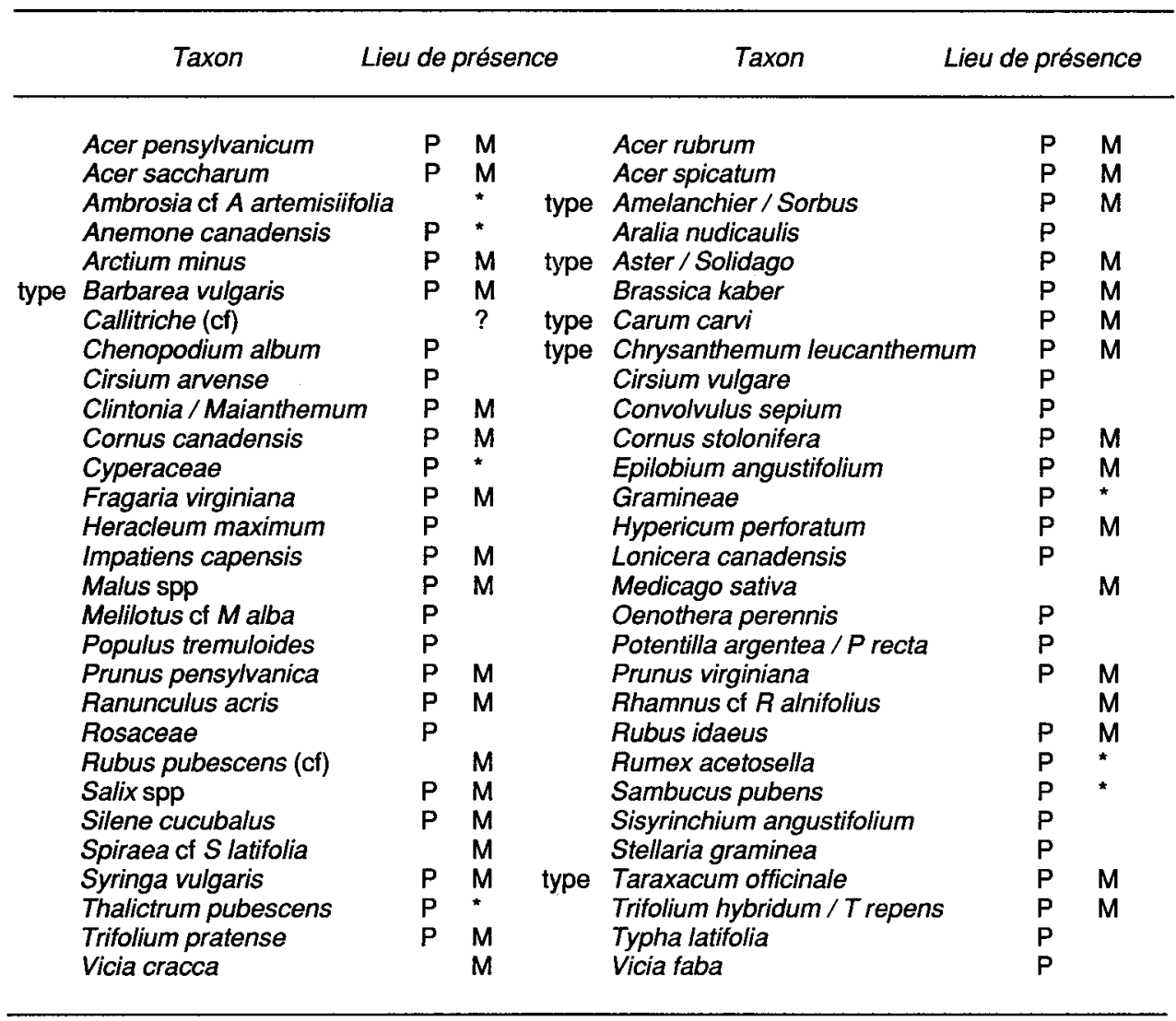

" : espèces anémophiles ou entomophiles sans nectaires floraux; ? : aucun renseignement sur le potentiel nectarifère.

\section{REMERCIEMENTS}

Nos remerciements s'adressent à Mme M Lassus, apicultrice, dont les connaissances apicoles et la constante collaboration ont grandement contribué au succès de ce projet. Nous remercions également le $\mathrm{Dr} C$ Lassus, MM $R$ Dionne, $\mathrm{C}$ Morneau et $\mathrm{C}$ Bertrand pour leur contribution. Cette recherche a été réalisée à l'aide de bourses octroyées par la DGES du ministère de l'Éducation du Québec, par le CRSNG du Canada et par le Fonds FCAR du Québec.

\section{Summary - Pollen and nectar sources} near Rimouski, Quebec, Canada. In 1980, the study of pollen yields and honeys from the same hive was conducted near Rimouski, in the Lower SaintLaurence River region (fig 1). Pollen yields were collected daily using a trap placed on a new colony. Half of the yields were examined through sorting of pellets (Louveaux, 1958a). Honey samples, covering one week each, were extracted at the end of the season using photographic compari- 
son of honeycombs. Standard meteorological data were recorded daily, and weekly surveys of flowering plants were performed within a $1.5 \mathrm{~km}$ radius around the apiary (fig 1).

A total of $1226 \mathrm{~g}$ of tresh pollen were extracted from the trap during a $3.5 \mathrm{mth}$ period (table I). Considering the trap efficiency, around $12.5 \mathrm{~kg}$ of fresh pollen were collected by the bees for a mean of $3.6 \mathrm{~kg} / \mathrm{mo}$. This result is remarkable, considering the restraints imposed on the colony and the bad weather conditions of 1980. On 60 taxa identified in the pellets (fig 2), only 16 displayed individual values greater than $1 \%$ of the total pollen crop (table II). The main pollen sources (> 10\%) were : Trifolium hybridum / $T$ repens, Cornus stolonifera, and Salix spp. Mixed pollen pellets were recorded in $65 \%$ of pollen yields but they represent only $0.4 \%$ of the total yield. Thirty-six (36) taxa were identified in mixed pellets (table III).

Pollen analysis of 13 successive honeys and 3 annual ones from the same apiary showed the presence of 42 taxa (fig 3). Other information about these honeys is given in table IV. Most of the honeys are poor in pollen grains. Honeys from the beginning of the season are mixed floral; they are replaced by Rubus idaeus monofloral honeys and then by mixed honeys preceding monofloral honeys of Trifolium hybridum / $T$ repens. The last successive honey presents a double dominance with Medicago sativa. Rubus and Trifolium association is also found in the 3 annual honeys. Pollen analysis of successive honeys has demonstrated the contamination of honey by pollen which is part of the bees diet. These phenomena, caused by the bees, would explain the presence in honey of species without floral nectaries and species which were no longer in bloom when the honey super was installed.
Most of the plants selected by the bees were present within $500 \mathrm{~m}$ of the apiary, were selected from the indigenous or naturalised flora and were visited for both pollen and nectar (table V).

honey / pollen pellett / pollen analysis / nectar plant / pollen plant / Canada

Zusammenfassung - Pollen- und Nek-
tartrachten bei Rimouski, Quebec, Kanada. Die Untersuchungen der Pollen-und Nektartrachten wurden im Jahr 1980 an ein und demselben Bienenvolk in SainteBlandine (Bezirk Rimouski) in der Region Lower Saint-Laurence River (Abb 1) durchgeführt. Die Polleneinträge wurden täglich mit Hilfe einer Polienfalle bestimmt. Die Hälfte der Erträge wurde durch Sortieren der Pollenhöschen bestimmt (Louveaux, 1958a). Die Honigproben von jeweils einer Woche wurden am Ende der Saison extrahiert und durch photografischen Vergleich der Honigwaben ausgewertet. Die standardmäßigen metereologischen Daten wurden täglich aufgezeichnet, die blühenden Pflanzen im Umkreis von 1,5 m (Radius) um den Bienenstand herum wurden wöchentlich protokolliert (Abb 1).

Insgesamt $1226 \mathrm{~g}$ frischer Pollen wurden über den Zeitraum von 3 1/2 Monaten hinweg der Falle entnommen (Tabelle I). Berücksichtigt man die Effizienz der Pollenfalle, so wurden also ca $12,5 \mathrm{~kg}$ frischer Pollen von den Bienen gesammelt, dh im Mittel $3,6 \mathrm{~kg}$ pro Monat. Dieses Ergebnis ist bemerkenswert, wenn man die Einschränkungen für das Volk und das schlechte Wetter im Jahr 1980 in Betracht zieht. Von 60 Taxa, die aus den Pollenhöschen identifiziert wurden (Abb 2), zeigten nur 16 eine höhere Frequenz als $1 \%$ des gesamten Pollenertrags (Tabelle II). Die hauptsächlichen Pollenspender (> 10\%) sind: Trifolium hybridum / $T$ repens, Cor- 
nus stolonifera und Salix spp. Gemischte Pollenhöschen wurden in $65 \%$ der Pollenproben gefunden, machten jedoch insgesamt nur $0,4 \%$ des Ertrags aus. 36 Taxa wurden aus den Mischpollenhöschen identifiziert (Tabelle III).

Die Pollenanalyse von 13 sukzessiven Honigen und 3 Jahreshonigen vom selben Bienenstand ergab 42 Taxa (Abb 3). Weitere Informationen über diese Honige sind in Tabelle IV aufgeführt. Die meisten Honige enthalten wenig Pollenkörner.

Die Honige am Anfang der Saison sind gemischtfloral, sie werden abgelöst durch monoflore Rubus idaeus Honige, dann folgen gemischtflorale und schließlich monoflorale Trifolium hybridum / $T$ repens Honige. Der letzte sukzessive Honig zeigt eine doppelte Dominanz mit Medicago sativa. Rubus und Trifolium. Gemische finden sich auch in den 3 Jahreshonigen. Die Pollenanalyse der sukzessiven Honige ergab auch eine Kontamination des Honigs mit Pollen aus der Bienennahrung. Dieses Phänomen, daß durch die Bienen verursacht wird, könnte das Vorkommen von Pollen in Honigen von Arten ohne florale Nektarien oder von Arten, die nicht mehr blühen, wenn der Honigraum aufgesetzt wird, erklären.

Die meisten Pflanzen, die von Bienen aufgesucht werden, werden wegen Pollen und Nektar besucht (Tabelle VII), stehen innerhalb von $500 \mathrm{~m}$ Umkreis um den Bienenstand und entstammen der lokalen einheimischen oder eingebürgerten Flora.

\section{Honig / Pollenhöschen / Pollenanalyse / Nektarpflanze / Pollenpflanze / Kanada}

\section{RÉFÉRENCES}

Adams RJ, Manville GC, McAndrews JH (1978) Comparison of Pollen Collected by a Honey Bee Colony with a Modern Wind-dispersed
Pollen Assemblage. Can Field Nat 92, 359368

Adams RJ, Smith MV, Townsend GF (1979) Identification of Honey Sources by Pollen Analysis of Nectar from the Hive. J Apic Res 18, 292-297

Betts AD (1920) The Constancy of the Pollencollecting Bee. Bee World 2, 10-11

Betts AD (1935) The Constancy of the PollenCollecting Bee. Bee World 16, 111-113

Birks HJB (1973) Pollen and Spore Identifications. In: Past and Present Vegetation of the Isle of Skye. A Palaeoecological Study. Cambridge Univ Press, Londres

Chabot JN (1948) Plantes mellifères du Québec. Minis Agric Québec, Serv Hortic (non publié)

DeGrandi-Hoffman G, Hoopingarner R, Klomparens $\mathrm{K}$ (1986) Influence of Honey Bee (Hymenoptera : Apidae) In-hive Pollen Transfer on Cross-pollination and Fruit Set in Apple. Environ Entomol 15, 723-725

Eckert JE (1942) The Pollen Required by a CoIony of Honeybees. J Econ Entomol 35, 309311

Environnement Canada $(1978,1979,1980)$ Résumé mensuel. Données météorologiques pour le Canada oriental. Service de l'Environnement atmosphérique, 63, 64, 65. Partie 3

Feller-Demalsy MJ (1983) Le spectre pollinique des miels du Québec. Apidologie 14, 147174

Feller-Demalsy MJ, Lamontagne $Y$ (1979) Analyse pollinique des miels du Québec. Apidologie 10, 313-340

Feller-Demalsy MJ, Parent J, Strachan AA (1987a) Microscopic analysis of honeys from Alberta, Canada. J Apic Res 26, 123-132

Feller-Demalsy MJ, Parent J, Strachan AA (1987b) Microscopic analysis of honeys from Saskatchewan, Canada. J Apic Res 26, 247254

Feller-Demalsy MJ, Parent J (1989a) Analyse pollinique des miels de l'Ontario, Canada. Apidologie 20, 127-138

Feller-Demalsy MJ, Parent J, Strachan AA (1989b) Microscopic analysis of honeys from Manitoba, Canada. J Apic Res 28, 41-49

Free JB (1963) The Flower Constancy of Honeybees. J Anim Ecol 32, 119-131 
Goltz LR (ed) (1977) Honey Plants. Gleanings in bee culture, Medina, Ohio

Hirschfelder H (1951) Quantitative Untersuchungen zum Polleneintragen der Bienenvölker. Z Bienenforsch 1, 67-77

Lamontagne $Y$ (1975) Composition chimique de pollens recueillis au rucher expérimental de Deschambault (mai 73 à août 74). Minist Agric Québec (non publié)

Louveaux J (1954) Études sur la récolte du pollen par les abeilles. Apiculteur (sect sci) 98, 43-50

Louveaux J (1955) Introduction à l'étude de la récolte du pollen par les abeilles (Apis mellifica L). Physiol Comp CEcol 4, 1-54

Louveaux J (1958a) Recherche sur la récolte du pollen par les abeilles (Apis mellifica L). Thèse de doctorat, Univ Paris

Louveaux J (1958b) Recherches sur l'origine dans le miel du pollen de plantes entomophiles dépourvues de nectaires. Ann Abeille 1, 89-92

Louveaux J (1966) Pollenanalyse einiger kanadischer Honige. Z Bienenforsch 8, 195-202

Louveaux J, Maurizio A, Vorwohl G (1978) International Commission for Bee Botany of IUBS. Methods of Melissopalynology. Bee World 59, 139-157

Maurizio A (1953) Weitere Untersuchungen an Pollenhöschen. Beih Schweiz Bienenztg 2, 485-556 (Résumé)

Maurizio A, Kollmann H (1949) Beobachtungen an Pollenhöschen. Beih Schweiz Bienenztg 2, 442-455 (Résumé)

McLellan AR (1976) Factors Affecting Pollen Harvesting by the Honeybee. J Appl Ecol 13, 801-811
Mitchener AV (1948) Nectar and Pollen Producing Plants in Manitoba. Sci Agric 28, 475480

Morse RA (1980) The Complete Guide to Beekeeping (rev ed). Dutton, New York

Murrell DC, Szabo TI (1981) Pollen Collection by Honey Bees At Beaverlodge, Alberta. Am Bee J 121, 885-888

Oertel E (1939) Honey and Pollen Plants of the United States. US Dep Agric, Washington DC, Circ 554

Oertel E (1967) Nectar and Pollen Plants. In: Beekeeping in the United States. US Dep Agric Handbook No 335

PPellett FC (1976) American Honey Plants (5e ed). Dadant, Hamilton, IL

Percival M (1947) Pollen Collection by Apis mellifera. New Phytol 46, 142-173

Ramsay J (1987) Plants for Beekeeping in Canada and the Northern USA. Int Bee Res Assoc, Londres

Robinson FA, Oertel E (1975) Sources of nectar and pollen. In: The Hive and the Honey Bee. Dadant and Sons, Hamilton, III

Smith MV, Adie A (1963) A New Design in Pollen Traps. Can Bee J 74, 4-5, 8

Soehngen $U$ (1971) Crop content and pollen load studies of honey bees. Thèse de doctorat, Université du Manitoba

Synge AD (1947) Pollen Collection by Honeybees (Apis mellifera). J Anim Ecol 16, 122138

Todd FE, Bishop RK (1940) Trapping Honeybee-Gathered Pollen and Factors Affecting Yields. J Econ Entomo/ 33, 866-870 